\title{
Characteristics of craniofacial morphology and factors affecting them in patients with isolated cleft palate
}

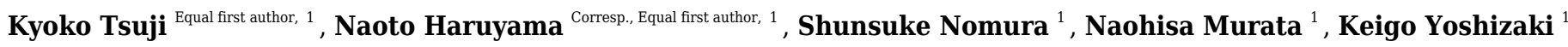 \\ , Takeshi Mitsuyasu ${ }^{2}$, Hiroyuki Nakano ${ }^{3}$, Seiji Nakamura $^{2}$, Yoshihide Mori $^{3}$, Ichiro Takahashi $^{1}$ \\ ${ }^{1}$ Section of Orthodontics and Dentofacial Orthopedics, Faculty of Dental Science, Kyushu University, Fukuoka, Fukuoka, Japan \\ 2 Section of Oral and Maxillofacial Oncology, Faculty of Dental Science, Kyushu University, Fukuoka, Fukuoka, Japan \\ 3 Section of Oral and Maxillofacial Surgery, Faculty of Dental Science, Kyushu University, Fukuoka, Fukuoka, Japan \\ Corresponding Author: Naoto Haruyama \\ Email address: haruyama@dent.kyushu-u.ac.jp
}

Background. Myriad maxillo-mandibular occlusal relationships are observed in patients with isolated cleft palate (ICP), unlike in patients with other cleft types, such as cleft lip and palate.

Objectives. This study aimed to categorise the characteristics of craniofacial morphology in patients with ICP, and investigate the clinical factors affecting these categorised morphological characteristics.

Methods. Thirty-six girls with ICP [age (mean \pm SD): $5.36 \pm 0.36$ years] underwent cephalometric measurement. Their craniofacial morphology was categorised using cluster analysis. Profilograms were created and superimposed onto the standard Japanese profilograms to visualise the morphological characteristics of each group (cluster). The mean values and variations in the linear and angular measurements of each group were compared with the Japanese standards and statistically analysed using Dunnett's test after the analysis of variance. Fisher's exact test was used to analyse the differences between the cleft types (cleft in the hard and/or soft palate) and skills of the operating surgeons in the groups.

Results. Cluster analysis of craniofacial morphologies in patients with ICP resulted in the formation of three categories: the first cluster exhibited a relatively harmonious anteroposterior relationship between the maxilla and the mandible (22.2\%); the second cluster exhibited crossbite owing to a significantly smaller maxilla (33.3\%); and the third cluster exhibited a smaller mandible with posterior rotation showing skeletal class II malocclusion (44.4\%). Differences in cleft types and surgeons were not associated with the distribution of patients in each cluster.

Conclusions. Patients with ICP exhibited characteristic morphological patterns, such as bimaxillary retrusion or severe mandibular retrusion, besides the anterior crossbite frequently found in patients with cleft lip and palate. Understanding the typical morphological characteristics could enable better diagnostic categorisation of patients with ICP, which may eventually improve orthodontic treatment planning. 


\section{Characteristics of craniofacial morphology and factors 2 affecting them in patients with isolated cleft palate}

3

4

5

22

23

24

25

26

27

28

29

30

31

32

33

34

35

36

37

38

39

40

41

Kyoko Tsuji ${ }^{1 *}$, Naoto Haruyama ${ }^{1 *}$, Shunsuke Nomura ${ }^{1}$, Naohisa Murata ${ }^{1}$, Keigo Yoshizaki ${ }^{1}$, Takeshi Mitsuyasu ${ }^{2}$, Hiroyuki Nakano ${ }^{3}$, Seiji Nakamura ${ }^{2}$, Yoshihide Mori ${ }^{3}$, Ichiro Takahashi ${ }^{1}$

${ }^{1}$ Section of Orthodontics and Dentofacial Orthopedics, Faculty of Dental Science, Kyushu University, Fukuoka-shi, Fukuoka, Japan

${ }^{2}$ Section of Oral and Maxillofacial Oncology, Faculty of Dental Science, Kyushu University, Fukuoka-shi, Fukuoka, Japan

${ }^{3}$ Section of Oral and Maxillofacial Surgery, Faculty of Dental Science, Kyushu University, Fukuoka-shi, Fukuoka, Japan

* Kyoko Tsuji and Naoto Haruyama should be considered joint first author.

Corresponding Author:

Naoto Haruyama ${ }^{1}$

3-1-1 Maidashi, Higashi-ku, Fukuoka-shi, Fukuoka, 812-8582, Japan

Email address: haruyama@dent.kyushu-u.ac.jp 
42

43 Abstract

44 Background. Myriad maxillo-mandibular occlusal relationships are observed in patients with 45 46 isolated cleft palate (ICP), unlike in patients with other cleft types, such as cleft lip and palate.

Objectives. This study aimed to categorise the characteristics of craniofacial morphology in patients with ICP, and investigate the clinical factors affecting these categorised morphological characteristics.

Methods. Thirty-six girls with ICP [age (mean \pm SD): $5.36 \pm 0.36$ years] underwent cephalometric measurement. Their craniofacial morphology was categorised using cluster analysis. Profilograms were created and superimposed onto the standard Japanese profilograms to visualise the morphological characteristics of each group (cluster). The mean values and variations in the linear and angular measurements of each group were compared with the Japanese standards and statistically analysed using Dunnett's test after the analysis of variance. Fisher's exact test was used to analyse the differences between the cleft types (cleft in the hard and/or soft palate) and skills of the operating surgeons in the groups.

Results. Cluster analysis of craniofacial morphologies in patients with ICP resulted in the formation of three categories: the first cluster exhibited a relatively harmonious anteroposterior relationship between the maxilla and the mandible (22.2\%); the second cluster exhibited crossbite owing to a significantly smaller maxilla (33.3\%); and the third cluster exhibited a smaller mandible with posterior rotation showing skeletal class II malocclusion (44.4 \%). Differences in cleft types and surgeons were not associated with the distribution of patients in each cluster.

Conclusions. Patients with ICP exhibited characteristic morphological patterns, such as bimaxillary retrusion or severe mandibular retrusion, besides the anterior crossbite frequently found in patients with cleft lip and palate. Understanding the typical morphological characteristics could enable better diagnostic categorisation of patients with ICP, which may eventually improve orthodontic treatment planning.

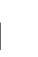

2


74

\section{Introduction}

76 Cleft lip and/or palate (CL/P) is one of the most common congenital anomalies in the orofacial 77 78 79 80 area (Schutte \& Murray, 1999). Patients with cleft palate including the isolated cleft palate (ICP) and the cleft lip and palate (CLP) generally undergo palatoplasty at the age of 1-1.5 years to obtain velopharyngeal competence and normal speech. The various palatoplasty techniques, such as von Langenbeck's method (Langenbeck, 1861), Veau-Wardill-Kilner push-back method (Wardill, 1937), intravelar veloplasty (Braithwaite \& Maurice, 1968), and Furlow double opposing Z-plasty (Furlow, 1986), have been introduced by different surgeons. Although there are many variations of these techniques, the scar formed on maxillary oral and nasal mucosa by palatoplasty is thought to impair maxillary growth, which is probably responsible for the high frequency of anterior crossbite in patients operated for cleft palate. For example, a survey of 996 Japanese patients with CLP (Kouno, Suzuki \& Watanabe, 1989) found that the reverse occlusion involving at least one tooth was observed in $84.6 \%$ of CLP cases. Vettore et al. also observed that the frequencies of anterior crossbite and posterior crossbite in Brazilian patients with CLP were $60.7 \%$ and $39.3 \%$, respectively (Vettore \& Sousa Campos, 2011). Sæle et al. demonstrated that $61.4 \%$ of Norwegian patients with CLP had Angle's class III malocclusion (Sæle et al., 2017).

However, the suppression of maxillary bone growth due to palatoplasty is not necessarily a problem in patients with ICP. Hermann et al. investigated unoperated patients with ICP and suggested that children with ICP had a shorter maxilla, reduced maxillary posterior height, shorter mandible, and reduced posterior height of the mandible with retrognathia, compared to controls with unilateral isolated cleft lip (ICL) (Hermann et al., 2002). On the other hand, Fujita et al. reported that the average maxillary length was shorter and the nasomaxillary complex was generally positioned more posteriorly in operated patients with ICP than in controls with normal occlusion; however, the craniofacial pattern exhibited a wide variation in patients operated for ICP (Fujita et al., 2005). Nakasone et al. observed a variety of mandibular configurations and jaw relationships, indicating the diversity of craniofacial morphologies in patients operated for ICP (Nakasone et al., 2013). These reports imply that the characteristics of craniofacial morphology are not sufficiently understood by simply comparing the cephalometric mean values 
104 between patients operated for ICP and controls, such as those with unilateral ICL or the normal 105 population.

106 The objective of this study was to categorise the characteristics of craniofacial morphology in 107 patients operated for ICP, to elucidate the manifestation patterns before orthodontic treatment, 108 and to investigate the causal relationships between morphological categories and potential key 109 factors, such as the differences in cleft type (range of cleft) and skills of the operating surgeons.

110

111

112

113

114

115

116

117

118

119

120

121

122

123

124

125

126

127

128

129

130

131

132

133

134

135

\section{Materials \& Methods}

\section{Sample and data collection}

This study protocol was reviewed and approved by the Kyushu University Institutional Review Board for Clinical Research (\#27-135). A dedicated website was established with detailed information on this clinical study in case patients wished to opt out of the investigation. For this retrospective cohort study, the participants were selected from among patients with ICP who first visited the Department of Orthodontics at Kyushu University Hospital between 2002 and 2014. The inclusion criterion was: patients with overt ICP (i.e., clefts of the hard and soft palate or clefts of the soft palate) $(n=77)$. The exclusion criteria were: patients with syndromes affecting the craniofacial morphology including the Pierre Robin sequence $(n=1)$, patients who underwent palatoplasty other than push-back method $(n=7)$, missing diagnostic records according to being transferred to other hospitals or the default of their appointment $(n=16)$, and incomplete diagnostic records (i.e., cephalogram taken with the mouth opened) or unwanted developmental stages (i.e., other than Hellman's dental stage IIA (Completion of the primary dentition by the acquisition of second deciduous molars) (Hellman, 1935)) $(\mathrm{n}=5)$. Of the 48 selected patients, 12 boys were excluded from the study sample because their numbers were not sufficient for the categorisation and comparison of craniofacial morphology. Thirty-six girls, who underwent lateral cephalography before orthodontic diagnosis, belonging to Hellman's dental stage IIA were eventually included in the study. The 36 patients were aged $5.36 \pm 0.36$ years (mean $\pm \mathrm{SD}$ ). The participants were evaluated based on the cleft type (range of cleft): 23 patients had clefts of the hard and soft palate and 13 had clefts of the soft palate. All primary surgeries were performed by a group of similarly trained oral surgeons, and using the modified Wardill's push-back method at $1.55 \pm 0.21$ years (mean $\pm \mathrm{SD}$ ) of age.

Peer) reviewing PDF | (2020:08:52363:2:0:NEW 8 Mar 2021) 
136

137

138

139

140

141

142

143

144

145

146

147

148

149

150

151

152

153

154

155

156

157

158

159

160

161

162

163

164

165

166

\section{Cephalometric measurements and cluster analysis}

All the cephalograms were taken on the same machine to account for the magnification factor.

The magnification factor in lateral cephalometric radiographs was $110 \%$ at the midsagittal plane. WinCeph 10.0 (Rise Corp., Sendai, Japan) was used to perform the cephalometric measurements, according to previous studies (Iizuka, 1958; Sakamoto, 1959), using the reference points represented in Figure 1. The explanations for all reference points are indicated in the legend.

All cephalometric measurements were conducted by the same orthodontist to eliminate interexaminer errors. All measurements were recorded again after two months. Dahlberg's formula (Dahlberg, 1940) was used to evaluate the reproducibility of the measurements. The measurement error (distance) of the coordinates of 17 points used in the profilogram (Sakamoto, 1959) was $0.61 \mathrm{~mm}$. The measurement error (angle) of the 10 representative angular measurements (Facial angle, Convexity, A-B plane, Y-axis, FH to SN, SNA, SNB, ANB, N-Pog to $\mathrm{SN}$, and Nasal floor to $\mathrm{SN}$ ) was $0.43^{\circ}$.

Cluster analysis was performed to categorise the characteristics of craniofacial morphology using JMP Pro14 (SAS Institute Inc. Cary, NC, USA). The variables used for cluster analysis were based on a previous study (Yamanouchi et al., 1995) and included the lower anterior facial height [anterior nasal spine to menton (ANS-Me)], length of the maxilla [point Apterygomaxillary fissure (A'-Ptm')], length of the mandible [condylion-gonion (Cd-Gn)], anteroposterior position of the maxilla [sella, nasion, point A (SNA)], anteroposterior relationship of the maxilla and mandible (ANB), facial profile (facial angle), mandibular shape (gonial angle), and mandibular rotation [ramus plane to the sella-nasion line (SN)]. The linear measurements were normalised based on the upper anterior facial height [nasion to ANS (NANS)] before the cluster analysis, to eliminate the effect of the differences in the size of each individual (Yamanouchi et al., 1995). A best-suited model of 3 clusters was obtained using the Ward's method, defining the distances between groups and establishing the least possible dispersion within groups to ensure the greatest homogeneity for each cluster (Fig. 2) (de FrutosValle et al., 2020).

Average profilograms of each cluster were obtained and superimposed over the standard Japanese age matched female profilogram (Sakamoto, 1959) to visualise the characteristics of craniofacial morphology of each cluster. Furthermore, the mean linear or angular measurements 
167 of each cluster were statistically compared to the Japanese female standard of linear

168 measurements (Sakamoto, 1959) or to the Japanese standard of angular measurements obtained 169 from combined male and female subjects (Iizuka, 1958), respectively.

170

\section{3. Statistical analysis}

172 Multiple comparisons were performed using Dunnett's test following an analysis of variance 173 (ANOVA) by Prism 7 (GraphPad Software, San Diego, CA, USA). Moreover, Fisher's exact test 174 was performed to determine whether the differences in cleft type (cleft in the soft palate and/or 175 hard palate) or surgeons (who performed the palatoplasty) affected the craniofacial morphology in each cluster by $\mathrm{R}$ (the R Foundation, https://www.r-project.org). Post-hoc power (1- $\beta$ ) analysis for the ANOVA and Fisher's exact test was performed by G*power 3.1 (Faul et al., 2009). $P<0.05$ was considered statistically significant.

\section{Results} analysis

Participants were categorised into the following three clusters based on the results of the cluster analysis: cluster A (n=8), cluster B (n=12), and cluster C (n=16) (Fig. 2).

Cluster A exhibited no difference in the maxillary position compared to the standard, when the average profilograms of each cluster were superimposed over the standard profilogram; however, the mandibular body and anterior facial height were larger than those in the standard profilogram. The anteroposterior relationship of the maxilla and mandible was harmonious in cluster A (Fig. 3A). Cluster B exhibited significant maxillary retrusion and a tendency towards lingual inclination of the lower incisors, a shorter mandibular ramus, and a larger gonial angle than those of the standard. Anterior crossbite was observed. (Fig. 3B). Cluster C exhibited mandibular and maxillary retrusion, large ramus inclination, and clockwise rotation of the mandible (Fig. 3C).

Superimposition of the profilograms of all clusters over the standard profilogram revealed that 196 the anterior-most maxillary position was observed the most in the standard, followed by that in 
197 clusters $\mathrm{A}, \mathrm{C}$, and $\mathrm{B}$, while the most anterior mandibular position was observed the most in

198 cluster A, followed by that in the standard, cluster B, and cluster C (Fig. 4).

199

200

\section{Quantitative assessment of facial morphology in each cluster}

201

202

203

204

205

206

207

208

209

210

211

212

213

214

215

216

217

218

219

220

221

222

223

224

225

226

227

The mean linear and angular measurements of each cluster were compared to their standard counterparts (Iizuka, 1958; Sakamoto, 1959) to clarify the morphological characteristics of each cluster (Tables 1 and 2). Cluster A exhibited a significantly longer $\mathrm{Gn}-\mathrm{Cd}$ (mandibular length) and larger A-B plane angle than the standard, and significantly smaller FH to SN and L1 to mandibular plane angles. These results indicate that the characteristics of cluster A included a larger mandible and slight lingual inclination of the lower incisors. Cluster B exhibited a significantly larger A-B plane and occlusal plane to FH, and significantly smaller SNA, L1 to mandibular plane, convexity, ANB, and anterior incisal height (Is-Is') than the standard. These results indicate that the characteristics of cluster B included a tendency towards a skeletal class III relationship with maxillary retrusion. Cluster C exhibited a significantly larger Y-axis, nasal floor to $\mathrm{SN}$, nasal floor to $\mathrm{FH}$, mandibular plane to $\mathrm{FH}$, ramus to $\mathrm{SN}$, ramus to $\mathrm{FH}$, occlusal plane to $\mathrm{SN}$, and occlusal plane to $\mathrm{FH}$, and a significantly smaller facial angle, SNB, and N-Pog to $\mathrm{SN}$ than the standard. These results indicate that the characteristics of cluster $\mathrm{C}$ included clockwise rotation of the occlusal unit, including the maxilla and mandible, resulting in a profile exhibiting mandibular retrusion with a skeletal class II relationship.

\section{Relationships between craniofacial morphology and the differences in cleft type and surgeons}

Patients with ICP in this study included those with clefts of the hard and soft palate and cleft of the soft palate only, and were operated on by four different surgeons (surgeons A-D). Thus, the respective relationships of craniofacial morphology with differences in cleft type and surgeons were also analysed. The results of Fisher's exact test for cleft type $\left[P\left(\chi^{2}\right)=0.27\right]$ indicated no bias in patients' distribution. The results of Fisher's exact test for surgeons $\left[P\left(\chi^{2}\right)=\right.$ 0.69 ] also did not indicate any bias. Taken together, these findings indicate that the morphological differences in ICP were not significantly affected by the cleft type or skills of the operating surgeon (Table 3). 


\section{Discussion}

229 Patients with ICP, unlike those with other cleft types (e.g. CLP), present with a wide variety of 230 maxillo-mandibular occlusal relationships (Fujita et al., 2005; Nakasone et al., 2013).

231 Understanding the typical morphological features is crucial for the correct orthodontic diagnosis 232 and treatment planning. Cluster analysis of craniofacial morphologies in patients with ICP in the 233 present study resulted in their categorisation into three groups: a cluster with a relatively 234 harmonious anteroposterior relationship between the maxilla and the mandible (22.2\%), a 235 cluster with crossbite caused by a significantly smaller maxilla (33.3\%), and a cluster with a 236 smaller mandible with posterior rotation showing skeletal class II relationship (44.4\%). These

237 238 239 240 241

242 243 results suggest a significant difference from the craniofacial morphology of patients with CLP.

$$
\text { We selected cluster analysis for categorising craniofacial morphologies in this study. Cluster }
$$
analysis is a method of collecting and classifying items with similar characteristics from a group containing a mixture of differing characteristics (Finkelstein, Lavelle \& Hassard, 1989). Cluster analysis was considered more suitable for categorising characteristics compared to other methods of analysis, which require prior information about the potential groups, owing to the diversity of craniofacial morphology in patients with ICP.

Several previous studies have used the principal component analysis to determine the variates to be used before conducting the cluster analysis of craniofacial morphologies (Bui et al., 2006; Uribe et al., 2013; de Frutos-Valle et al., 2020). However, it is necessary to understand the clinical significance of the principal components, and sometimes it is even difficult to understand the significance of obtained principal components. Therefore, the variates used for cluster analysis in this study were based on Yamanouchi et al.'s study, which reported that the characteristics of facial morphology were well characterised by linear measurements of the lower anterior facial height (ANS-Me) and lengths of the maxilla and mandible (A'-Ptm' and Cd-Gn) and angular measurements including the anteroposterior position of the maxilla (SNA), anteroposterior relationship of the maxilla and mandible (ANB), facial profile (facial angle), mandibular shape (gonial angle), and mandibular rotation (ramus to SN) (Yamanouchi et al., 1995). They also reported that these skeletal factors were not significantly affected by local dental factors.

Soma reported that the differences in the absolute sizes of the jaw or face makes it difficult to compare craniofacial patterns simultaneously and that isometric processing (normalisation of 
259 linear measurements) is an effective method for the analysis of craniofacial patterns (Soma, 260 1977). In the present study, normalisation of linear measurements was achieved by dividing the 261 values with the anterior facial height (N-ANS), which is one of the most reliable measurements. 262 This normalization is important to eliminate the effect of individual variation in absolute length 263 on the cluster analysis (i.e., a larger individual may have a greater absolute length compared to a 264 smaller individual when, in fact, the length may be smaller if normalized for size). We believe 265 that the normalisation of linear measurements successfully eliminated the influence of size and 266 better clarified the trends in the characteristics of each sample.

267 Determination of the number of clusters is subjective and can result in variability between 268 studies. We deemed it appropriate to classify the participants into three cluster groups, which 269 could be understood easily without over-segmentation, based on the dendrogram created by 270 cluster analysis using the Ward's method (Fig. 2). The comparison of the mean values of each 271 cluster (obtained from cephalometric analysis) with the standard values showed that the large 272 mandibular size in cluster A was compensated (dentally) by a slight lingual inclination of the 273 lower incisors. Cluster B had a retruded maxilla and lingual inclination of the lower incisors, 274 which were similar to the dental compensation observed in cluster A. Cluster $\mathrm{C}$ had a retruded 275 mandible with clockwise rotation of the palatal plane, occlusal plane, mandibular plane, and 276 ramus plane. These results, based on cephalometric analyses, were faithfully represented in the 277 superimposed profilograms. Clusters A, B, and C constituted $22.2 \%, 33.3 \%$, and $44.4 \%$ of the 278 total study population, respectively, suggesting that several patients with ICP have mandibular 279 hypoplasia (retruded mandible; cluster C), in whom the suppression of maxillary growth post280 palatoplasty would not lead to major disharmony in the anteroposterior relationship of the jaw.

281 We speculated that the craniofacial morphology in patients with ICP may have formed 282 congenitally or acquired by mechanisms that differ from those in patients with CLP because the 283 craniofacial morphological characteristics of patients with ICP differ from those of patients with 284 CLP, who often exhibit anterior crossbite (Kouno, Suzuki \& Watanabe, 1989; Vettore \& Sousa 285 Campos, 2011; Sæle et al., 2017).

286 Several studies that compared ICP with ICL (as the control group) found that patients with ICP 287 had smaller mandibles than those of the controls (Hermann et al., 2002; Eriksen et al., 2006), 288 which strongly indicates a link between mandibular hypoplasia occurring during mandibular 289 development and ICP. Price et al. reviewed 930 papers including clinical and basic research on 
290 the relationship between cleft palate and mandibular hypoplasia, and concluded that mandibular 291 hypoplasia triggers cleft palate formation (Price, Haddad \& Fakhouri, 2016). Pierre Robin 292 sequence is characterised by a spectrum of anatomical anomalies including mandibular 293 hypoplasia, glossoptosis, life-threatening obstructive apnoea, and feeding difficulties. Typically, 294 a wide U-shaped cleft palate is associated with Pierre Robin sequence (Lehman, Fishman \& 295 Neiman, 1995); however, a narrow V-shaped cleft is associated with other cleft types. From this 296 perspective, there could be two important causes of ICP: primary failure of palatal fusion 297 (similar to other cleft types), and secondary failure of palatal fusion owing to a small mandible as 298 299 seen in Pierre Robin sequence. A previous study suggested that the width of the cleft at the posterior end of the hard palate and the total length of the cleft are significantly related to the severity of Pierre Robin sequence (Godbout et al., 2014). Unfortunately, the cleft shape or width before palatal closure could not be evaluated in our study because our surgical records were not tailored for the purpose of this study. Therefore, the relationships between the cleft shape/width before surgery and craniofacial morphology could not be determined. Indeed, we did not find any patients with typical manifestations of Pierre Robin sequence such as glossoptosis, life305 threatening obstructive apnoea, and feeding difficulties in their earliest infancy in this study. However, patients with mild Pierre Robin sequence cannot be completely distinguished from those with ICP with a small mandible. Therefore, it is conceivable that the number of participants with a relatively wide U-shaped cleft by mild Pierre Robin sequence may have affected the distribution of patients in the three categorised groups.

In a previous study, compensatory growth of the mandible was not observed at least during the first 2 years of life in patients with Pierre Robin sequence (Hermann et al., 2003). Moreover, 312 none of the patients with Pierre Robin sequence exhibited significant improvements in the 313 skeletal pattern that could be construed as a gradual correction of the initial severe skeletal class 314 II relationship after the age of 5 years (Daskalogiannakis, Ross \& Tompson, 2001). These studies 315 also support the notion that this sampling age would be suitable for identifying the typical 316 morphological characters of ICP, even if a certain number of patients with mild Pierre Robin 317 sequence are included in the study.

318 Nakasone et al. found that while the average severity of maxillary growth suppression was 319 mild in patients with ICP, individual cases displayed a wide variety of facial morphologies, from 320 mandibular protrusion to maxillary protrusion, and that averaging the characteristics of several 
321 cases resulted in values that resembled the standard value (Nakasone et al., 2013). They also 322 indicated that although the mean value of measurements related to the mandible was similar to 323 the standard value, a large variation was found in mandibular measurements, suggesting the 324 existence of a wide variety of mandibular shapes or positions and maxillo-mandibular 325 relationships in patients with ICP. Therefore, although patients with ICP often present with 326 mandibular hypoplasia at birth, they can present a wide variety of phenotypes, possibly due to 327 the additive effect of the acquired influence of palatoplasty.

328 One of the limitations of this report is that only girls were selected for the present study. 329 Previous studies have shown that ICP is more common in girls than in boys (Mossey et al., 2009; 330 Martelli et al., 2012). Similarly, fewer boys with ICP were found than girls with this condition 331 between 2002 and 2014 during the study selection process, i.e. 12 boys and 36 girls. Therefore, 332 boys were excluded from the study sample because their numbers were not sufficient for the 333 categorisation and comparison of craniofacial morphology. Moreover, because of the gender 334 difference in incidence in the ICP, there might be gender-specific aetiology that would affect 335 336 craniofacial morphology of ICP, suggesting that a group consisting both genders would be inappropriate to be analysed. It will be necessary to examine whether craniofacial morphologies of boys with ICP are in line with the results of the present study.

Based on the appropriate statistical approach, we believe that the results obtained are clinically useful for understanding the typical morphological characteristics of ICP. However, we admit that the retrospective cohort with a relatively small sample may slightly undermine the results. Further evaluation of the prospective cohort study with a large sample would be warranted.
A recent systematic review of the effects of functional appliances suggested that the skeletal changes elicited by orthodontic treatment may be negligible or statistically insignificant (Cacciatore et al., 2019). The ANB angle increased more effectively over a short period of time in the treatment of skeletal class III malocclusion using maxillary protraction during the growth period, compared to that in the untreated group; however, the effect become less pronounced toward the end of growth period and the skeletal pattern became almost similar to that of the untreated control group (Vaughn et al., 2005; Mandall et al., 2010, 2012, 2016). These reports suggest that the skeletal pattern is not significantly altered by orthodontic treatment or residual growth at least in participants without CLP or ICP. However, we selected younger patients in Hellman's stage IIA before they underwent orthodontic intervention because we wished to 
352 exclude the effects of orthodontic treatment on the skeletal pattern and thus on the determination

353 of typical morphological characteristics of ICP, which are encountered at the beginning of the

354 orthodontic treatment. Further studies with samples after growth complete will be required for

355 better evaluation and understanding of craniofacial morphology of ICP.

356 The present study population included patients with clefts of the soft palate and those with

357 clefts of the hard and soft palate. Surgeries for clefts of the soft palate are often less invasive

358 because they require lesser removal of mucosa from the nasal side than that required for clefts of

359 the hard and soft palate. Thus, this research was also aimed at confirming whether the

360 differences in the cleft type affect the degree of maxillary growth suppression. Moreover, the

361 differences between the skills of the four surgeons were also considered because they may affect

362 maxillary development. Table 3 shows the sample distribution for each category. Fisher's exact

363 test was used to examine the distribution of the small sample size. The data in Table 3A and B

364 exhibit $P$-values greater than 0.05 and relatively high power $(1-\beta)$, indicating that the distribution

365 was not significantly different. These results suggest that differences in the cleft type (cleft

366 range) and skills of the operating surgeon were not significantly associated with the craniofacial

367 morphology of patients with ICP. Therefore, the characteristics of three clusters obtained from

368 this ICP population can be expected to represent the typical craniofacial morphologies

369 encountered in the clinical practice, which reflect the characteristics of ICP itself and the effects

370 of any subsequent surgeries. This information could be beneficial in orthodontic treatment

371 planning for patients with cleft palate.

372

\section{Conclusions}

374 Cluster analysis of craniofacial morphologies in patients with ICP resulted in the identification of

375 three clusters: a cluster with a relatively harmonious anteroposterior relationship between the

376 maxilla and the mandible; that with anterior crossbite caused by a significantly small maxilla;

377 and that with a smaller mandible with posterior rotation showing a skeletal class II relationship.

378 A total of $44.4 \%$ of the patients were classified into the cluster characterised by a skeletal class

379 II relationship with a smaller mandible (cluster C). Differences in the cleft type (cleft range) and

380 skills of the surgeon were not associated with the distribution of craniofacial morphology. These

381 results suggest that the mechanisms responsible for craniofacial morphology of patients with ICP

382 are different from those in patients with CLP. Understanding the typical morphological 
383 characteristics could enable better diagnostic categorisation of patients with ICP, which may

384 eventually improve orthodontic treatment planning.

385

386

\section{Acknowledgements}

388

389

390

391

392

393

394

395

396

397

398

399

400

401

402

403

404

405

406

407

408

409

410

411

412

We wish to thank all the members of our department for participating in useful discussions on the topic of the study.

\section{References}

Braithwaite F, Maurice DG. 1968. The importance of the levator palati muscle in cleft palate closure. British journal of plastic surgery 21:60-2. DOI: 10.1016/s0007-1226(68)80087-6.

Bui C, King T, Proffit W, Frazier-Bowers S. 2006. Phenotypic characterization of Class III patients. The Angle orthodontist 76:564-9. DOI: 10.1043/00033219(2006)076[0564:PCOCIP]2.0.CO;2.

Cacciatore G, Ugolini A, Sforza C, Gbinigie O, Plüddemann A. 2019. Long-term effects of functional appliances in treated versus untreated patients with Class II malocclusion: A systematic review and meta-analysis. PLoS ONE 14:1-26. DOI: 10.1371/journal.pone.0221624.

Dahlberg G. 1940. Statistical Methods for Medical and Biological Students. London: Allen and Unwin.

Daskalogiannakis J, Ross RB, Tompson BD. 2001. The mandibular catch-up growth controversy in Pierre Robin sequence. American Journal of Orthodontics and Dentofacial Orthopedics 120:280-285. DOI: 10.1067/mod.2001.115038.

Eriksen J, Hermann N, Darvann T, Kreïborg S. 2006. Early postnatal development of the mandible in children with isolated cleft palate and children with nonsyndromic Robin sequence. Cleft Palate-Craniofacial Journal 43:160-167. DOI: 10.1597/04-113.1.

Faul F, Erdfelder E, Buchner A, Lang A-G. 2009. Statistical power analyses using G*Power 3.1: tests for correlation and regression analyses. Behavior research methods 41:1149-60. DOI: 10.3758/BRM.41.4.1149. 
413 Finkelstein M, Lavelle CL, Hassard T. 1989. The role of cluster analysis on traditional 414 cephalometric dimensions. The Angle orthodontist 59:97-106. DOI: 10.1043/0003$4153219(1989) 059<0097:$ TROCAO $>2.0 . C O ; 2$.

416 de Frutos-Valle L, Martin C, Alarcón JA, Palma-Fernández JC, Ortega R, Iglesias-Linares A. 417 2020. Sub-clustering in skeletal class III malocclusion phenotypes via principal component 418 analysis in a southern European population. Scientific Reports 10:1-12. DOI: $419 \quad 10.1038 / \mathrm{s} 41598-020-74488-\mathrm{w}$.

420 Fujita S, Suzuki A, Nakamura N, Sasaguri M, Kubota Y, Ohishi M. 2005. Retrospective 421 Evaluation of Craniofacial Growth of Japanese Children with Isolated Cleft Palate: From 422 Palatoplasty to Adolescence. Cleft Palate-Craniofacial Journal 42:625-632. DOI: $423 \quad 10.1597 / 032-163.1$.

424 Furlow LT. 1986. Cleft palate repair by double opposing Z-plasty. Plastic and reconstructive 425 surgery 78:724-38. DOI: 10.1097/00006534-198678060-00002.

426 Godbout A, Leclerc JE, Arteau-Gauthier I, Leclerc LD. 2014. Isolated versus Pierre Robin 427 sequence cleft palates: Are they different? Cleft Palate-Craniofacial Journal 51:406-411. 428 DOI: $10.1597 / 12-261$.

429 Hellman M. 1935. The face in its developmental career. Dental Cosmos 77:685-699.

430 Hermann N, Kreiborg S, Darvann T, Jensen B, Dahl E, Bolund S. 2002. Early Craniofacial 431 Morphology and Growth in Children with Unoperated Isolated Cleft Palate. Cleft Palate432 Craniofacial Journal 39:604-622. DOI: 10.1597/1545433 1569_2002_039_0604_ecmagi_2.0.co_2.

434 Hermann N V., Kreiborg S, Darvann TA, Jensen BL, Dahl E, Bolund S. 2003. Craniofacial 435 Morphology and Growth Comparisons in Children with Robin Sequence, Isolated Cleft 436 437 Palate, and Unilateral Complete Cleft Lip and Palate. The Cleft Palate-Craniofacial Journal 40:373-396. DOI: 10.1597/1545-1569_2003_040_0373_cmagci_2.0.co_2.

Iizuka T. 1958. Roentgencephalometric analysis of craniofacial growth in Japanese children. Journal of Stomatological Society Japan 25:260-272.

Kouno K, Suzuki A, Watanabe M. 1989. Clinical statistics on patients with cleft lip and/or palate for 19 years in the orthodontic clinic, Kyushu University. Journal of Japanese Cleft Palate Association 14:159-170. 
443 Langenbeck von B. 1861. Operation der anageborene totalen Spaltung des harten Gauments $444 \quad$ nach einer Methode. Dtsch. Arch. Klin Med. 13:231.

445 Lehman JA, Fishman JRA, Neiman GS. 1995. Treatment of Cleft Palate Associated with Robin 446 Sequence: Appraisal of Risk Factors. The Cleft Palate-Craniofacial Journal 32:25-29. 447 DOI: 10.1597/1545-1569_1995_032_0025_tocpaw_2.3.co_2.

448 Mandall NA, Cousley R, DiBiase A, Dyer F, Littlewood S, Mattick R, Nute S, Doherty B, 449 Stivaros N, McDowall R, Shargill I, Ahmad A, Walsh T, Worthington H. 2012. Is early class III protraction facemask treatment effective?Amulticentre, randomized, controlled trial: 3-Year follow-up. Journal of Orthodontics 39:176-185. DOI:

Mandall N, Cousley R, DiBiase A, Dyer F, Littlewood S, Mattick R, Nute SJ, Doherty B, Stivaros N, McDowall R, Shargill I, Worthington H V. 2016. Early class III protraction facemask treatment reduces the need for orthognathic surgery: a multi-centre, two-arm parallel randomized, controlled trial. Journal of Orthodontics 43:164-175. DOI:

\subsection{0/14653125.2016.1201302.}

Mandall N, DiBiase A, Littlewood S, Nute S, Cousley R, Dyer F, Mattick R, Doherty B, Stivaros N, McDowall R, Shargill I, Worthington H. 2010. Is early class III protraction facemask treatment effective? A multicentre, randomized, controlled trial: 15-month follow-up. Journal of Orthodontics 37:149-161. DOI: 10.1179/14653121043056.

Martelli DRB, Machado RA, Swerts MSO, Rodrigues LAM, de Aquino SN, Martelli Júnior H. 2012. Non sindromic cleft lip and palate: Relationship between sex and clinical extension. Brazilian Journal of Otorhinolaryngology 78:116-120. DOI: 10.5935/18088694.20120018.

Mossey PA, Little J, Munger RG, Dixon MJ, Shaw WC. 2009. Cleft lip and palate. The Lancet 374:1773-1785. DOI: 10.1016/S0140-6736(09)60695-4.

Nakasone A, Susami T, Uchino N, Inokuchi T, Okayasu M, Uwatoko K, Takahashi N, Ohkubo K, Mori Y, Takato T. 2013. Craniofacial morphology of patients with isolated cleft palate before orthodontic treatment. Journal of Japanese Cleft Palate Association 38:113-119. cleft palate: A systematic review. Cleft Palate-Craniofacial Journal 53:e34-e44. DOI: 10.1597/14-238. 
474 Sæle P, Østhus E, Ådalen S, Nasir EF, Mustafa M. 2017. Pattern of clefts and dental anomalies

475 in six-year-old children: a retrospective observational study in western Norway. Acta

476 Odontologica Scandinavica 75:100-105. DOI: 10.1080/00016357.2016.1260770.

477 Sakamoto T. 1959. A study on the developmental changes of dentofacial complex of Japanese

478 with special reference to Sella Turcica. Journal of Japanese Orthodontic Society 18:1-17.

479 Schutte BC, Murray JC. 1999. The many faces and factors of orofacial clefts. Human molecular

480 genetics 8:1853-9. DOI: $10.1093 / \mathrm{hmg} / 8.10 .1853$.

481 Soma K. 1977. Graphic display and isometric pattern analysis for craniofacial growth. Journal of 482 Japanese Orthodontic Society 36:316-322.

483 Uribe LMM, Vela KC, Kummet C, Dawson D V., Southard TE. 2013. Phenotypic diversity in 484 white adults with moderate to severe Class III malocclusion. American Journal of

485 Orthodontics and Dentofacial Orthopedics 144:32-42. DOI: 10.1016/j.ajodo.2013.02.019.

486 Vaughn GA, Mason B, Moon HB, Turley PK. 2005. The effects of maxillary protraction therapy

487 with or without rapid palatal expansion: A prospective, randomized clinical trial. American

488 Journal of Orthodontics and Dentofacial Orthopedics 128:299-309. DOI:

$489 \quad$ 10.1016/j.ajodo.2005.04.030.

490 Vettore M V., Sousa Campos AE. 2011. Malocclusion characteristics of patients with cleft lip 491 and/or palate. The European Journal of Orthodontics 33:311-317. DOI:

$492 \quad 10.1093 /$ ejo/cjq078.

493 Wardill WEM. 1937. The technique of operation for cleft palate. British Journal of Surgery 494 25:117-130. DOI: 10.1002/bjs.1800259715.

495 Yamanouchi S, Ishihara K, Shirato Y, Sato K, Mitani H. 1995. Facial pattern of the present day 496 Japanese associated with normal occlusion. Journal of Japanese Orthodontic Society $497 \quad 54: 93-101$.

498

499

500 Figure legends

501 Fig. 1 Reference points for the cephalometric measurements

502 (A) N: nasion, the anterior-most point of the frontonasal suture; S: sella turcica, the estimated 503 centre of the hypophyseal fossa; Or: orbitale, the deepest point on the infraorbital margin; Po: 504 porion, the upper margin of the ear canal; Ba: basion, the anterior-most margin of the foramen 
505 magnum; Cd; condylion, the most supradorsal point of the condylar head; Ar: articulare, the 506 intersection between the external contour of the cranial base and dorsal contour of the condylar 507 head and neck; Ptm: pterygomaxillary fissure, the inferior point of the fissure; PNS: posterior 508 nasal spine; ANS: anterior nasal spine; A: subspinale, the deepest point on the premaxilla 509 between the ANS and prosthion in the midline; U1 (Is) and U1a : upper incisor constructed 510 between the incisal tip of the anterior-most deciduous maxillary central incisor and its apex; Mo:

511 mid-point of the deciduous maxillary second molar; L1 (Ii) and L1a: lower incisor constructed

512 between the incisal tip of the most anterior deciduous mandibular central incisor and its apex;

513 Pog: pogonion, the anterior-most point of the bony chin; B: supramentale, the posterior-most

514 point in the concavity between the infradentale and Pog; Me: menton, the lowest point on the

515 symphyseal shadow; Gn: gnathion, the point on the chin determined by bisecting the angle

516 formed by the facial plane and mandibular plane; Go: gonion, the intersection between the ramus

517 plane and the mandibular plane; A': intersection of a perpendicular drawn from point $A$ to the

518 palatal plane; Ptm': intersection of a perpendicular line from the Ptm to the palatal plane; Ms':

519 intersection of the perpendicular drawn from Mo to the palatal plane;

520 palatal plane: the square dotted line; mandibular plane: the dashed line

521 (B) U1 (Is): upper incisor constructed between the incisal tip of the most anteriorly placed

522 deciduous maxillary central incisor; Is': intersection of the perpendicular drawn from Is to the 523 palatal plane; L1 (Ii): lower incisor constructed between the incisal tip of the most anteriorly 524 placed deciduous mandibular central incisor; Ii': intersection of the perpendicular drawn from Ii 525 to the palatal plane; Mo: mid-point of the deciduous maxillary second molar; Ms': intersection of 526 the perpendicular drawn from Mo to the palatal plane; Mi': intersection of the perpendicular 527 drawn from Mo to the mandibular plane;

528 palatal plane: the square dotted line; mandibular plane: the dashed line 529

530 Fig. 2 Dendrogram created by cluster analysis using Ward's method

531 Thirty-six girls were classified into three groups, i.e. clusters A, B, and C.

532

533 Fig. 3 Averaged profilograms of each cluster and their superimposition over the Japanese 534 standard profilogram 
535 Points S, N, Or, ANS, A, U1, L1, B, Pog, Me, Go, Ar, PNS, and Mo were connected on the 536 profilograms to visualise the facial pattern [according to a previous study (Sakamoto, 1959)].

537 The superimposition was performed at point S, parallel to the Frankfurt horizontal (FH) plane.

538 The square dotted line, dashed line, dash-dotted line, and solid line represent clusters A, B, C, 539 and the Japanese standard, respectively. (A) Cluster A exhibited no difference in maxillary 540 position compared to the standard. The mandible and maxilla showed a balanced anteroposterior

541 relationship. (B) Cluster B exhibited significant maxillary retrusion and tendencies towards

542 lingual inclination of the lower incisors, a shorter mandibular ramus, and a larger gonial angle,

543 with crossbite. (C) Cluster C exhibited mandibular and maxillary retrusion, a larger ramus angle,

544 and clockwise rotation of the mandible.

545 The ANB in the averaged profilograms of clusters $\mathrm{A}, \mathrm{B}$, and $\mathrm{C}$ was $+3.1^{\circ},+0.7^{\circ}$, and $+4.8^{\circ}$, 546 respectively.

547 Abscissa: FH-parallel line through S; Ordinate: FH-perpendicular line through S.

548 ANS, anterior nasal spine; A, subspinale; S, sella turcica; N, nasion; Or, orbitale; U1, incisor tip

549 of the most anteriorly placed deciduous maxillary central incisor; L1, lower incisor tip of the

550 most anteriorly placed deciduous mandibular central incisor; B, supramentale; Pog, pogonion;

551 Me, menton; Go, gonion; Ar, articulare; PNS, posterior nasal spine; Mo, mid-point of the

552 deciduous maxillary second molar

553

554 Fig. 4 Superimposition of all the clusters over the Japanese standard profilogram

555 The superimposition was performed at point S, parallel to the Frankfurt horizontal (FH) plane.

556 The square dotted line, dashed line, dash-dotted line, and solid line represent clusters A, B, C,

557 and the Japanese standard, respectively.

558 Abscissa: FH-parallel line through S; Ordinate: FH-perpendicular line through S.

559 S: sella turcica

560

561

562 Table 1 Comparison between the mean angular measurements of the Japanese standard and each 563 cluster

564 Table 2 Comparison between the mean linear measurements of the Japanese standard and each 565 cluster 
566 Table 3 Relationships of craniofacial morphology with the differences in cleft type and surgeons 567 A) Patient distribution according to the cleft type

568 B) Patient distribution according to the operating surgeon 


\section{Figure 1}

Reference points for the cephalometric measurements

(A) N: nasion, the anterior-most point of the frontonasal suture; S: sella turcica, the estimated centre of the hypophyseal fossa; Or: orbitale, the deepest point on the infraorbital margin; Po: porion, the upper margin of the ear canal; Ba: basion, the anterior-most margin of the foramen magnum; Cd; condylion, the most supradorsal point of the condylar head; Ar: articulare, the intersection between the external contour of the cranial base and dorsal contour of the condylar head and neck; Ptm: pterygomaxillary fissure, the inferior point of the fissure; PNS: posterior nasal spine; ANS: anterior nasal spine; A: subspinale, the deepest point on the premaxilla between the ANS and prosthion in the midline; U1 (Is) and U1a : upper incisor constructed between the incisal tip of the anterior-most deciduous maxillary central incisor and its apex; Mo: mid-point of the deciduous maxillary second molar; L1 (li) and L1a: lower incisor constructed between the incisal tip of the most anterior deciduous mandibular central incisor and its apex; Pog: pogonion, the anterior-most point of the bony chin; B: supramentale, the posterior-most point in the concavity between the infradentale and Pog; Me: menton, the lowest point on the symphyseal shadow; Gn: gnathion, the point on the chin determined by bisecting the angle formed by the facial plane and mandibular plane; Go: gonion, the intersection between the ramus plane and the mandibular plane; $A^{\prime}$ : intersection of a perpendicular drawn from point $A$ to the palatal plane; Ptm': intersection of a perpendicular line from the Ptm to the palatal plane; Ms': intersection of the perpendicular drawn from Mo to the palatal plane; palatal plane: the square dotted line; mandibular plane: the dashed line (B) U1 (Is): upper incisor constructed between the incisal tip of the most anteriorly placed deciduous maxillary central incisor; Is': intersection of the perpendicular drawn from Is to the palatal plane; L1 (li): lower incisor constructed between the incisal tip of the most anteriorly placed deciduous mandibular central incisor; li': intersection of the 
perpendicular drawn from li to the palatal plane; Mo: mid-point of the deciduous maxillary second molar; Ms': intersection of the perpendicular drawn from Mo to the palatal plane; Mi': intersection of the perpendicular drawn from Mo to the mandibular plane; palatal plane: the square dotted line; mandibular plane: the dashed line 
A

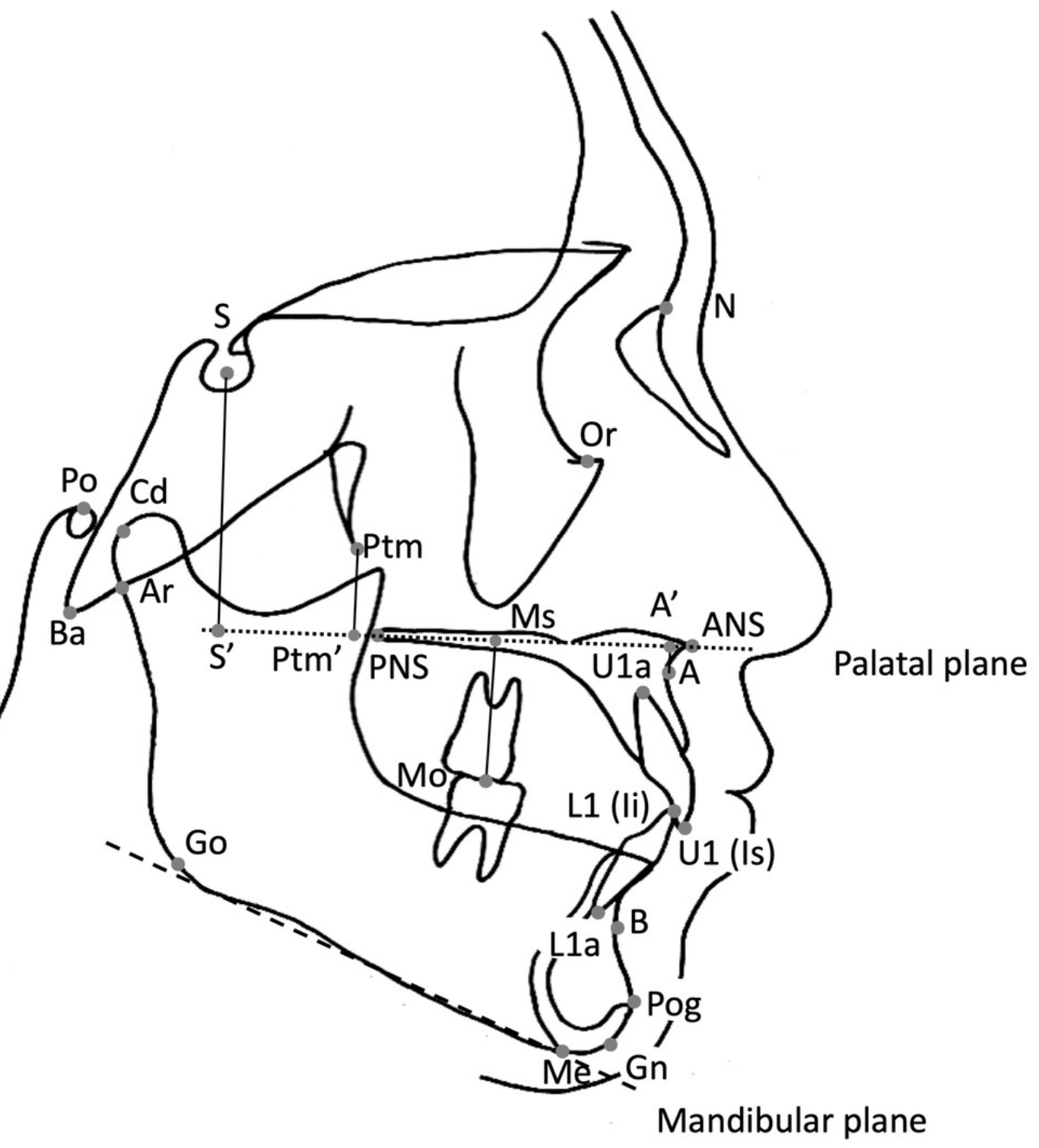

B

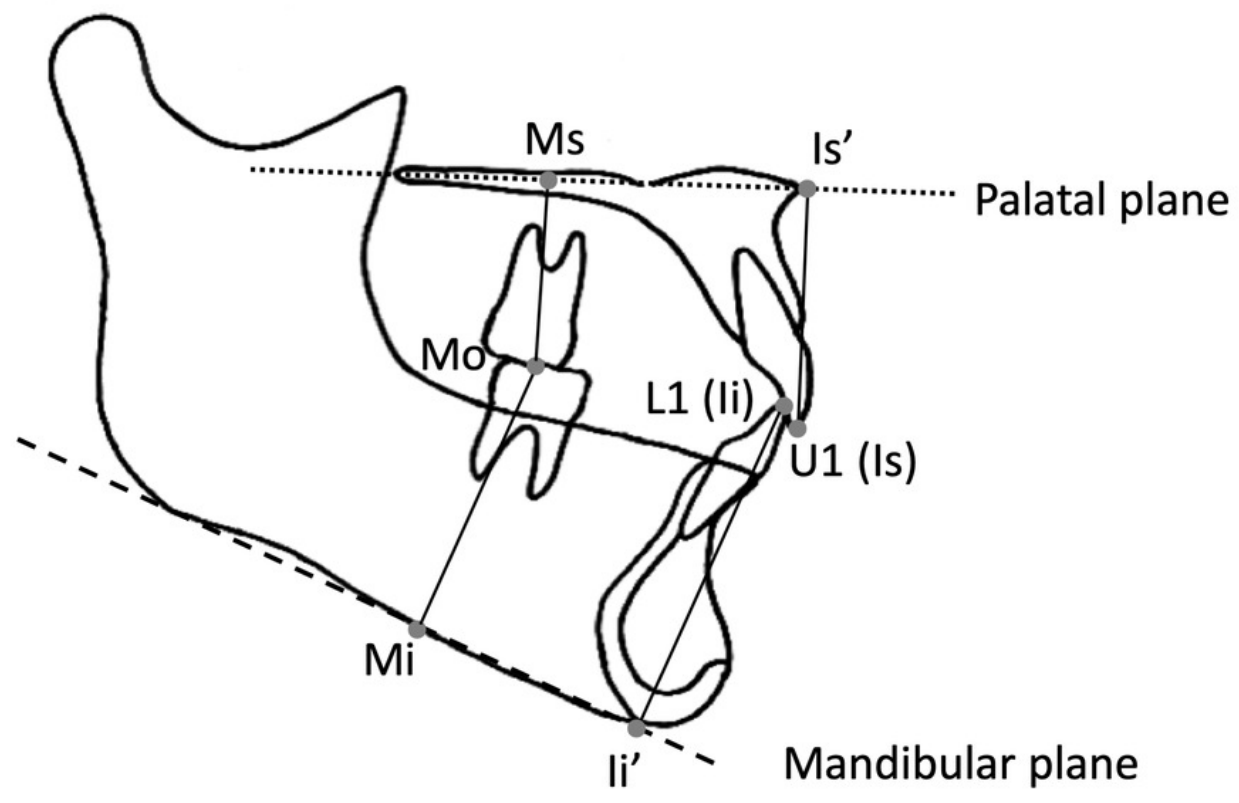


Figure 2

Dendrogram created by cluster analysis using Ward's method

Thirty-six girls were classified into three groups, i.e. clusters A, B, and C. 


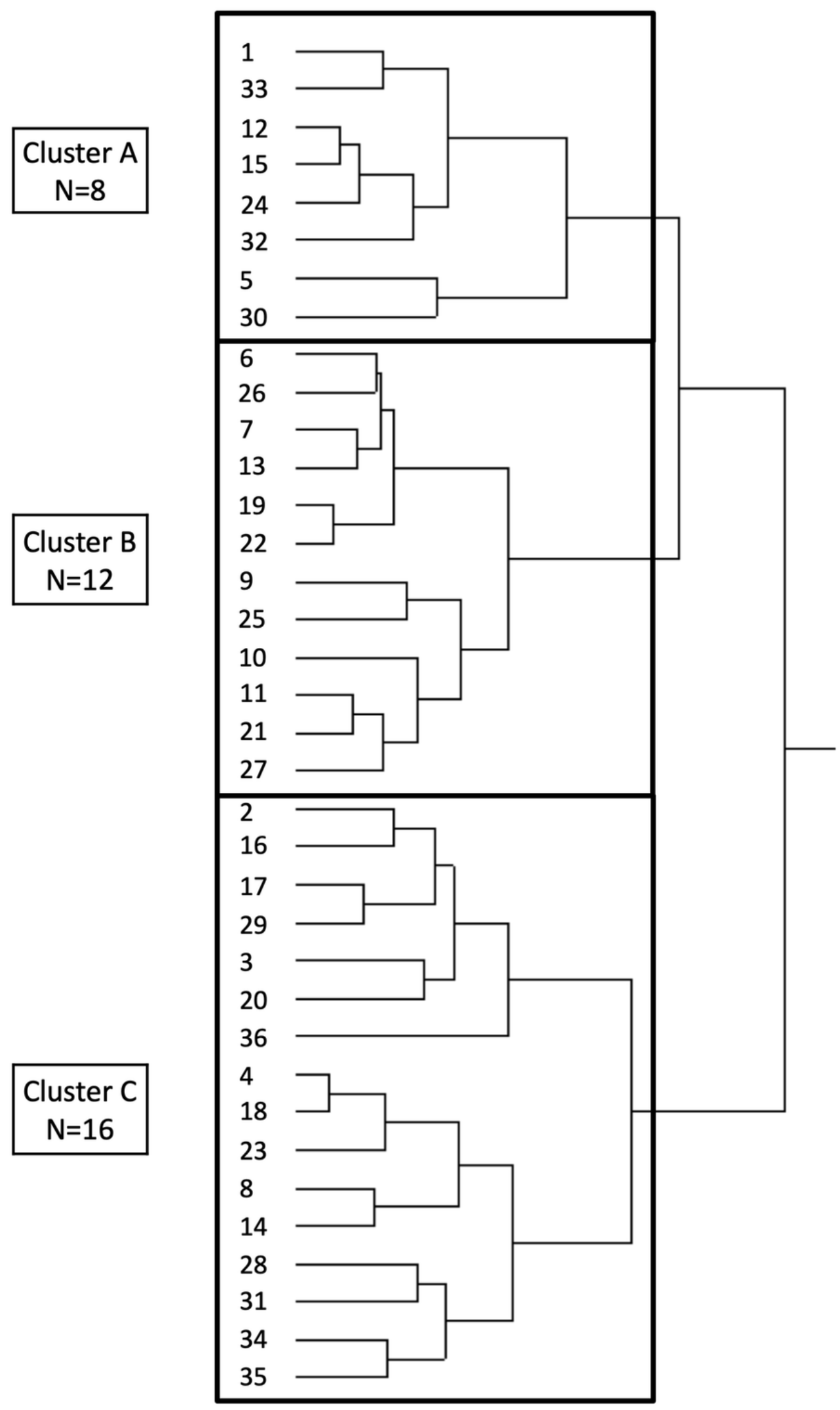




\section{Figure 3}

Averaged profilograms of each cluster and their superimposition over the Japanese standard profilogram

Points S, N, Or, ANS, A, U1, L1, B, Pog, Me, Go, Ar, PNS, and Mo were connected on the profilograms to visualise the facial pattern [according to a previous study (Sakamoto, 1959)

]. The superimposition was performed at point S, parallel to the Frankfurt horizontal (FH) plane. The square dotted line, dashed line, dash-dotted line, and solid line represent clusters A, B, C, and the Japanese standard, respectively. (A) Cluster A exhibited no difference in maxillary position compared to the standard. The mandible and maxilla showed a balanced anteroposterior relationship. (B) Cluster B exhibited significant maxillary retrusion and tendencies towards lingual inclination of the lower incisors, a shorter mandibular ramus, and a larger gonial angle, with crossbite. (C) Cluster $\mathrm{C}$ exhibited mandibular and maxillary retrusion, a larger ramus angle, and clockwise rotation of the mandible. The ANB in the averaged profilograms of clusters $A, B$, and $C$ was $+3.1^{\circ},+0.7^{\circ}$, and $+4.8^{\circ}$, respectively. Abscissa: FH-parallel line through S; Ordinate: FH-perpendicular line through S. ANS, anterior nasal spine; A, subspinale; S, sella turcica; N, nasion; Or, orbitale; U1, incisor tip of the most anteriorly placed deciduous maxillary central incisor; L1, lower incisor tip of the most anteriorly placed deciduous mandibular central incisor; B, supramentale; Pog, pogonion; Me, menton; Go, gonion; Ar, articulare; PNS, posterior nasal spine; Mo, mid-point of the deciduous maxillary second molar 


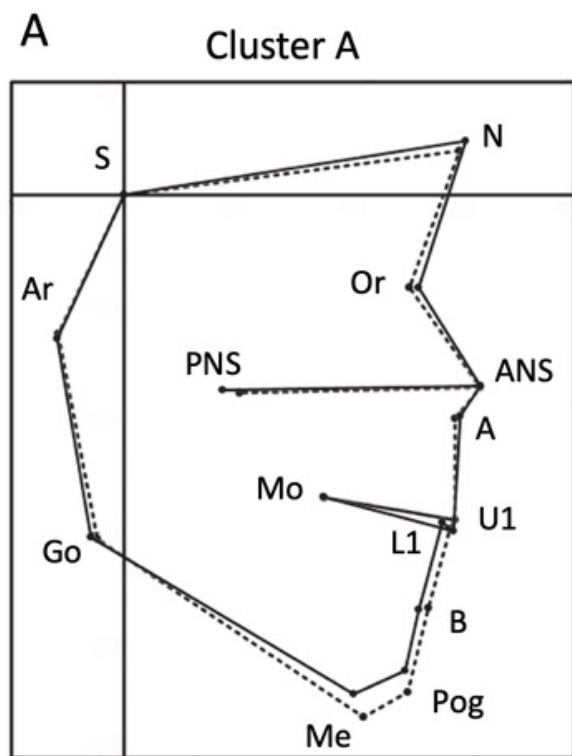

$\mathrm{ANB}=3.1^{\circ}$
B

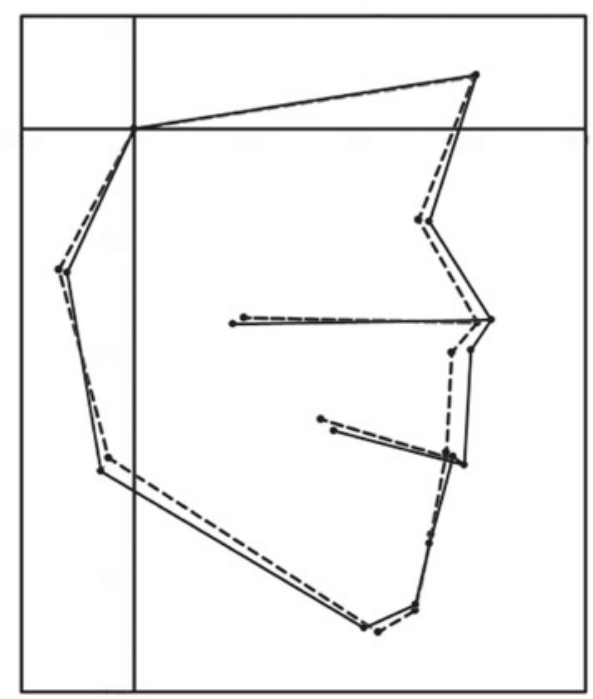

$\mathrm{ANB}=0.7^{\circ}$
C Cluster C

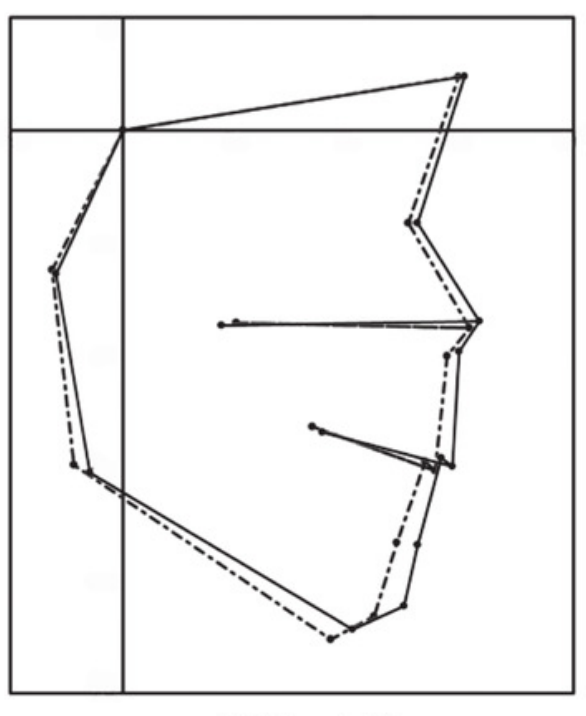

$\mathrm{ANB}=4.8^{\circ}$

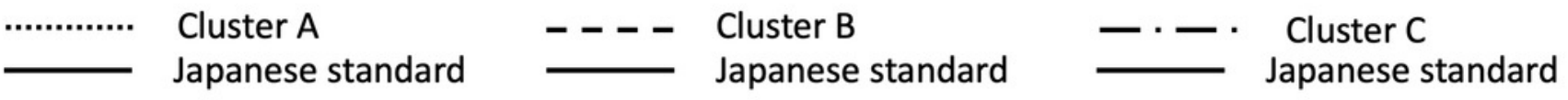




\section{Figure 4}

Superimposition of all the clusters over the Japanese standard profilogram

The superimposition was performed at point S, parallel to the Frankfurt horizontal (FH) plane.

The square dotted line, dashed line, dash-dotted line, and solid line represent clusters A, B,

$\mathrm{C}$, and the Japanese standard, respectively. Abscissa: FH-parallel line through S; Ordinate:

FH-perpendicular line through S. S: sella turcica 


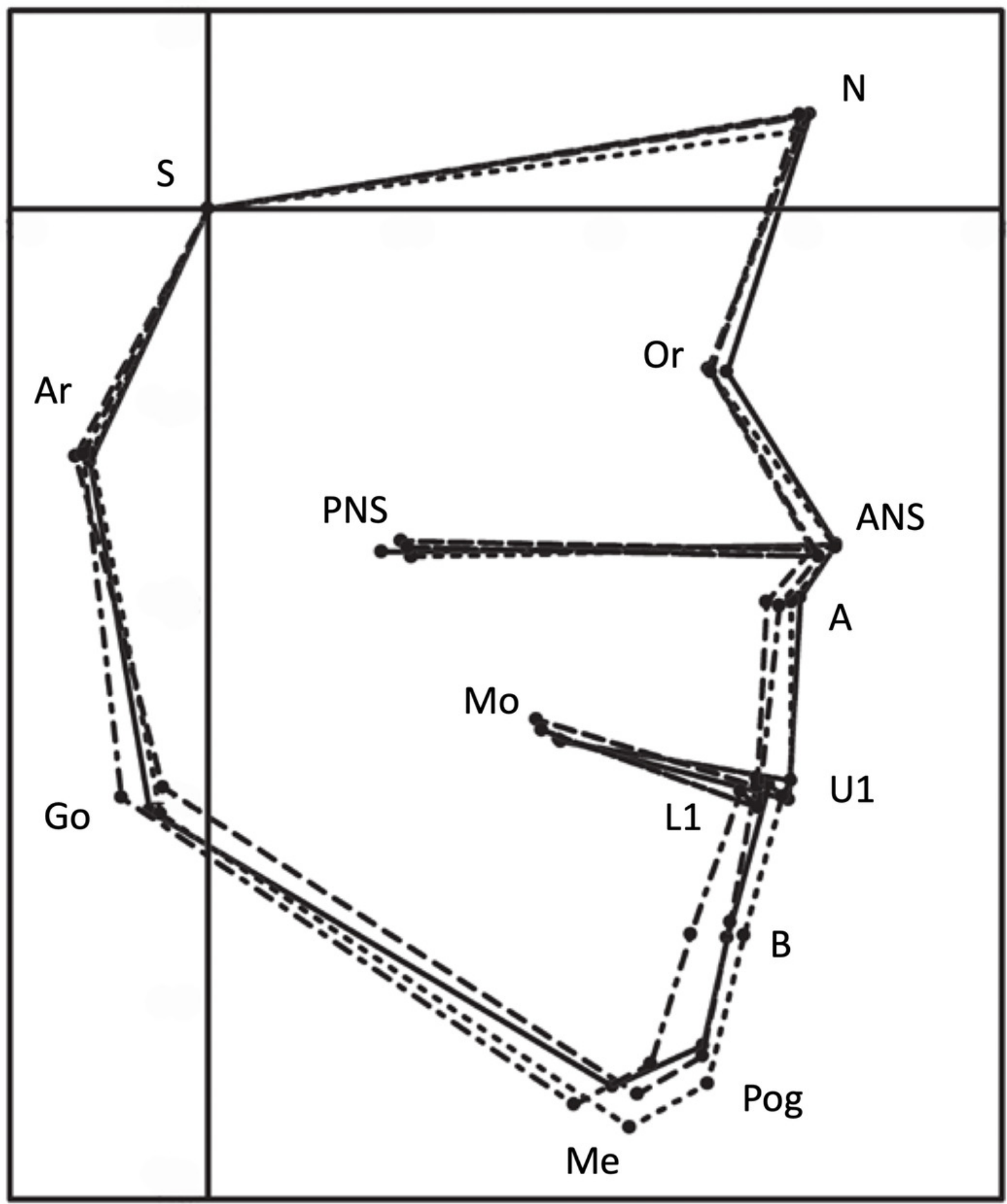

............ Cluster A

- - - Cluster B

-. - C Cluster C

— Japanese standard 


\section{Table $\mathbf{1}$ (on next page)}

Comparison between the mean angular measurements of the Japanese standard and each cluster

${ }^{*} P<0.05,{ }^{* * P}<0.01$ by Dunnett' s multiple comparison test. SD, standard deviation. 
1 Table 1 Comparison between the mean angular measurements of the Japanese standard and each cluster

\begin{tabular}{|c|c|c|c|c|c|c|c|c|c|c|c|c|c|}
\hline \multirow[b]{2}{*}{$\begin{array}{c}\text { Angular } \\
\text { measurements }\left(^{\circ}\right)\end{array}$} & \multicolumn{2}{|c|}{ Japanese standard } & \multicolumn{4}{|c|}{ Cluster A } & \multicolumn{3}{|c|}{ Cluster B } & \multicolumn{3}{|c|}{ Cluster C } & \multirow[b]{2}{*}{$\begin{array}{c}\text { Post-hoc } \\
\text { power } \\
(1-\beta)\end{array}$} \\
\hline & $\operatorname{Mean}( \pm$ SD) & $95 \%$ CI & Mea & $( \pm \mathbf{S D})$ & $95 \%$ CI & $P$-value & Mean $( \pm$ SD) & $95 \%$ CI & $P$-value & Mean $( \pm$ SD) & $95 \%$ CI & $P$-value & \\
\hline Facial angle & $84.5 \quad( \pm 3.2)$ & (83.3 to 85.7 ) & 84.5 & $( \pm 1.3)$ & (83.5 to 85.6$)$ & 1.000 & $83.7 \quad( \pm 1.3)$ & (82.9 to 84.5$)$ & 0.737 & $81.8 \quad( \pm 3.0)$ & (80.2 to 83.4$)$ & $0.005^{* *}$ & 0.620 \\
\hline Convexity & $\begin{array}{ll}10.6 & ( \pm 2.7) \\
\end{array}$ & (9.6 to 11.6$)$ & 8.9 & $( \pm 5.9)$ & (4.0 to 13.9 ) & 0.664 & $\begin{array}{|ll|}3.3 & ( \pm 5.9) \\
\end{array}$ & $(-0.5$ to 7.0$)$ & $0.000^{* *}$ & $11.9 \quad( \pm 4.8)$ & (9.3 to 14.5$)$ & 0.656 & 1.000 \\
\hline A-B plane & $-5.7 \quad( \pm 2.4)$ & $(-6.6$ to -4.8$)$ & -2.7 & $( \pm 2.7)$ & $(-5.0$ to -0.5$)$ & $0.032^{*}$ & $-0.1 \quad( \pm 4.1)$ & $(-2.7$ to 2.5$)$ & $0.000^{* *}$ & $-5.9 \quad( \pm 2.7)$ & $(-7.4$ to -4.4$)$ & 0.993 & 1.000 \\
\hline Y-axis & $61.5( \pm 3.4)$ & (60.2 to 62.8$)$ & 62.9 & $( \pm 1.6)$ & (61.5 to 64.2$)$ & 0.548 & $62.1 \quad( \pm 1.9)$ & (60.9 to 63.3$)$ & 0.906 & $64.6 \quad( \pm 3.5)$ & (62.7 to 66.4$)$ & $0.006^{* *}$ & 0.674 \\
\hline FH to $\mathrm{SN}$ & $10.1( \pm 3.0)$ & (9.0 to 11.2$)$ & 7.5 & $( \pm 1.3)$ & (6.4 to 8.6$)$ & $0.039^{*}$ & $8.7 \quad( \pm 2.1)$ & (7.4 to 10.1 ) & 0.300 & $9.5 \quad( \pm 2.5)$ & (8.1 to 10.8 ) & 0.760 & 0.450 \\
\hline$\angle \mathrm{SNA}$ & $80.1 \quad( \pm 3.4)$ & (78.8 to 81.4 ) & 81.6 & $( \pm 3.5)$ & (78.7 to 84.5$)$ & 0.502 & $76.6 \quad( \pm 2.6)$ & (74.9 to 78.3 ) & $0.005^{* *}$ & $78.1 \quad( \pm 2.7)$ & (76.7 to 79.6 ) & 0.121 & 0.890 \\
\hline$\angle \mathrm{SNB}$ & $76.0 \quad( \pm 3.5)$ & (74.7 to 77.3 ) & 78.5 & $( \pm 1.5)$ & (77.3 to 79.8 ) & 0.091 & $75.9 \quad( \pm 1.9)$ & (74.7 to 77.1 ) & 0.999 & $73.3 \quad( \pm 2.8)$ & (71.8 to 74.8 ) & $0.012^{*}$ & 0.842 \\
\hline$\angle \mathrm{ANB}$ & $4.1 \quad( \pm 3.5)$ & (2.8 to 5.4$)$ & 3.1 & $( \pm 2.3)$ & $(1.2$ to 5.0$)$ & 0.716 & $0.7 \quad( \pm 2.6)$ & $(-0.9$ to 2.3$)$ & $0.003^{* *}$ & $4.8 \quad( \pm 2.1)$ & (3.7 to 5.9$)$ & 0.754 & 0.769 \\
\hline N-Pog to SN & $75.3 \quad( \pm 3.8)$ & (73.9 to 76.8 ) & 77.0 & $( \pm 1.0)$ & (76.2 to 77.8 ) & 0.348 & $75.0 \quad( \pm 1.7)$ & (73.9 to 76.1 ) & 0.977 & $72.3 \quad( \pm 2.6)$ & (70.9 to 73.7 ) & $0.006^{* *}$ & 0.736 \\
\hline Nasal floor to SN & $7.7 \quad( \pm 3.6)$ & (6.3 to 9.1 ) & 6.2 & $( \pm 2.1)$ & (4.4 to 7.9 ) & 0.412 & $10.0 \quad( \pm 1.9)$ & (8.8 to 11.2 ) & 0.061 & $10.8 \quad( \pm 2.2)$ & (9.7 to 12.0 ) & $0.003^{* *}$ & 0.850 \\
\hline Nasal floor to FH & $-1.0 \quad( \pm 3.5)$ & $(-2.3$ to 0.3$)$ & -1.3 & $( \pm 2.7)$ & $(-3.6$ to 0.9$)$ & 0.988 & $1.3 \quad( \pm 2.4)$ & $(-0.2$ to 2.8$)$ & 0.083 & $1.4 \quad( \pm 2.6)$ & (0.0 to 2.8$)$ & $0.035^{*}$ & 0.604 \\
\hline $\begin{array}{l}\text { Mandibular plane to } \\
\text { SN }\end{array}$ & $39.2( \pm 4.4)$ & $(37.5$ to 40.9$)$ & 39.9 & $( \pm 3.1)$ & (37.4 to 42.5$)$ & 0.956 & $40.4 \quad( \pm 4.4)$ & (37.6 to 43.1$)$ & 0.785 & $42.3 \quad( \pm 5.0)$ & (39.6 to 45.0$)$ & 0.073 & 0.430 \\
\hline $\begin{array}{l}\text { Mandibular plane to } \\
\text { FH }\end{array}$ & $29.5 \quad( \pm 3.4)$ & (28.2 to 30.8$)$ & 32.4 & $( \pm 3.2)$ & (29.7 to 35.1$)$ & 0.230 & $31.6 \quad( \pm 4.4)$ & (28.8 to 34.4 ) & 0.353 & $32.9 \quad( \pm 6.0)$ & (29.7 to 36.1$)$ & $0.041^{*}$ & 0.825 \\
\hline Ramus plane to $\mathrm{SN}$ & $89.2 \quad( \pm 5.5)$ & (87.1 to 91.3 ) & 89.0 & $( \pm 4.6)$ & (85.2 to 92.9$)$ & 0.999 & $86.0 \quad( \pm 2.2)$ & (84.6 to 87.4$)$ & 0.104 & $94.9 \quad( \pm 3.3)$ & (93.1 to 96.6$)$ & $0.000^{* *}$ & 0.965 \\
\hline Ramus plane to FH & $79.9 \quad( \pm 5.2)$ & (77.9 to 81.9 ) & 81.5 & $( \pm 4.3)$ & (77.9 to 85.2$)$ & 0.673 & $77.3 \quad( \pm 2.9)$ & (75.5 to 79.1$)$ & 0.201 & $85.4 \quad( \pm 3.5)$ & (83.6 to 87.2$)$ & $0.000^{* *}$ & 0.956 \\
\hline Gonial angle & $130.0( \pm 5.3)$ & $(128.0$ to 132.0$)$ & 130.9 & $( \pm 7.3)$ & (124.8 to 137$)$ & 0.963 & $134.3( \pm 4.8)$ & (131.3 to 137.4$)$ & 0.081 & $127.5( \pm 6.3)$ & (124.1 to 130.8$)$ & 0.355 & 0.795 \\
\hline U1 to $\mathrm{SN}$ & $87.2 \quad( \pm 6.5)$ & (84.7 to 89.7$)$ & 92.0 & $( \pm 7.7)$ & (85.6 to 98.4 ) & 0.209 & $89.1 \quad( \pm 6.2)$ & (85.2 to 93.1$)$ & 0.759 & $85.2 \quad( \pm 7.8)$ & (81.0 to 89.3 ) & 0.672 & 0.532 \\
\hline U1 to $F H$ & $96.6 \quad( \pm 6.5)$ & (94.1 to 99.1 ) & 99.5 & $( \pm 6.9)$ & (93.8 to 105.2 ) & 0.592 & $97.9 \quad( \pm 6.6)$ & (93.7 to 102.0$)$ & 0.908 & $94.6 \quad( \pm 7.7)$ & (90.5 to 98.7$)$ & 0.677 & 0.304 \\
\hline $\begin{array}{l}\text { L1 to mandibular } \\
\text { plane }\end{array}$ & $85.7 \quad( \pm 4.1)$ & (84.1 to 87.3 ) & 78.9 & $( \pm 4.4)$ & (75.2 to 82.6$)$ & $0.041^{*}$ & $78.2 \quad( \pm 7.5)$ & (73.4 to 83.0 ) & $0.006^{* *}$ & $82.0( \pm 10.4)$ & (76.4 to 87.5 ) & 0.200 & 1.000 \\
\hline Interincisal angle & $148.4( \pm 9.5)$ & (144.8 to 152$)$ & 149.2 & $( \pm 11.2)$ & (139.9 to 158.6$)$ & 0.994 & $152.3( \pm 7.9)$ & $(147.3$ to 157.3$)$ & 0.538 & $150.5( \pm 11.8)$ & (144.3 to 156.8$)$ & 0.837 & 0.151 \\
\hline Occlusal plane to SN & $22.3( \pm 3.9)$ & (20.8 to 23.8 ) & 21.6 & $( \pm 2.1)$ & (19.9 to 23.3 ) & 0.914 & $24.0( \pm 3.0)$ & (22.1 to 25.9 ) & 0.342 & $26.9( \pm 3.1)$ & (25.2 to 28.6$)$ & $0.000^{* *}$ & 0.932 \\
\hline Occlusal plane to FH & $12.6( \pm 3.0)$ & (11.5 to 13.7$)$ & 14.1 & $( \pm 1.9)$ & (12.5 to 15.7$)$ & 0.490 & $15.2( \pm 3.2)$ & (13.2 to 17.3$)$ & $0.044^{*}$ & $17.5 \quad( \pm 3.7)$ & (15.5 to 19.4$)$ & $0.000^{* *}$ & 0.996 \\
\hline
\end{tabular}

$2 * P<0.05, * * P<0.01$ by Dunnett' s multiple comparison test. SD, standard deviation. 95\% CI, 95\% Confidence Interval. 


\section{Table 2 (on next page)}

Comparison between the mean linear measurements of the Japanese standard and each cluster

${ }^{*} P<0.05,{ }^{* * P}<0.01$ by Dunnett' s multiple comparison test. SD, standard deviation. 
1 Table 2 Comparison between the mean linear measurements of the Japanese standard and each cluster

\begin{tabular}{|c|c|c|c|c|c|c|c|c|c|c|c|c|c|c|c|c|}
\hline \multirow{3}{*}{\begin{tabular}{|c|}
$\begin{array}{c}\text { Linear } \\
\text { measurements } \\
(\mathbf{m m})\end{array}$ \\
$\mathrm{N}-\mathrm{S}$ \\
\end{tabular}} & \multicolumn{3}{|c|}{ Japanese standard } & \multicolumn{4}{|c|}{ Cluster A } & \multicolumn{4}{|c|}{ Cluster B } & \multicolumn{4}{|c|}{ Cluster C } & \multirow[b]{2}{*}{$\begin{array}{c}\text { Post-hoc } \\
\text { power } \\
(1-\beta)\end{array}$} \\
\hline & \multicolumn{2}{|c|}{ Mean ( \pm SD) } & \multirow{2}{*}{\begin{tabular}{|c|}
$\mathbf{9 5} \%$ CI \\
$(60.2$ to 62.8$)$ \\
\end{tabular}} & \multicolumn{2}{|c|}{ Mean $( \pm$ SD) } & \multirow{2}{*}{\begin{tabular}{|c|}
$\mathbf{9 5} \%$ CI \\
$(57.3$ to 63.1$)$ \\
\end{tabular}} & \multirow{2}{*}{\begin{tabular}{|c|}
$\boldsymbol{P}$-value \\
0.515 \\
\end{tabular}} & \multicolumn{2}{|c|}{ Mean $( \pm$ SD) } & \multirow{2}{*}{$\begin{array}{c}\mathbf{9 5} \% \mathbf{C I} \\
\text { (59.9 to } 62.2) \\
\end{array}$} & \multirow{2}{*}{\begin{tabular}{|c|}
$\boldsymbol{P}$-value \\
0.940 \\
\end{tabular}} & \multicolumn{2}{|c|}{ Mean ( \pm SD) } & \multirow{2}{*}{\begin{tabular}{c|}
$\mathbf{9 5 \%} \mathbf{C I}$ \\
(59.2 to 62.0$)$ \\
\end{tabular}} & \multirow{2}{*}{\begin{tabular}{|c|}
$\boldsymbol{P}$-value \\
0.673 \\
\end{tabular}} & \\
\hline & 61.5 & $( \pm 2.3)$ & & 60.2 & $( \pm 3.5)$ & & & 61.0 & $( \pm 1.8)$ & & & 60.6 & $( \pm 2.6)$ & & & 0.245 \\
\hline N-ANS & 43.7 & $( \pm 2.9)$ & (42.0 to 45.4$)$ & 42.2 & $( \pm 2.0)$ & (40.6 to 43.9$)$ & 0.344 & 43.7 & $( \pm 1.3)$ & (42.9 to 44.6$)$ & 1.000 & 44.8 & $( \pm 2.4)$ & (43.5 to 46.0$)$ & 0.440 & 0.368 \\
\hline ANS-Me & 58.9 & $( \pm 3.3)$ & (57.0 to 60.8$)$ & 62.2 & $( \pm 4.0)$ & (58.9 to 65.6$)$ & 0.127 & 57.8 & $( \pm 3.5)$ & (55.6 to 60.1$)$ & 0.813 & 60.2 & $( \pm 4.2)$ & (57.9 to 62.4$)$ & 0.689 & 0.739 \\
\hline N-Me & 100.0 & $( \pm 4.2)$ & (97.6 to 102.4$)$ & 102.1 & $( \pm 5.2)$ & (97.7 to 106.4$)$ & 0.629 & 100.2 & $( \pm 4.3)$ & $(97.5$ to 102.9$)$ & 0.999 & 102.3 & $( \pm 5.1)$ & (99.6 to 105.0) & 0.407 & 0.345 \\
\hline S'-Ptm' & 16.9 & $( \pm 2.1)$ & (15.7 to 18.1$)$ & 16.8 & $( \pm 2.5)$ & (14.7 to 18.9$)$ & 0.998 & 17.4 & $( \pm 1.4)$ & (16.5 to 18.3$)$ & 0.823 & 17.6 & $( \pm 1.5)$ & (16.8 to 18.4$)$ & 0.593 & 0.149 \\
\hline $\mathbf{A}^{\prime}-\mathbf{P t m}$ & 41.9 & $( \pm 2.1)$ & (40.7 to 43.1$)$ & 41.1 & $( \pm 2.5)$ & (39.1 to 43.2$)$ & 0.798 & 39.8 & $( \pm 1.9)$ & (38.6 to 41.0$)$ & 0.061 & 41.0 & $( \pm 2.6)$ & (39.6 to 42.4$)$ & 0.573 & 0.665 \\
\hline Ptm'-Ms & 17.6 & $( \pm 2.1)$ & (16.4 to 18.8$)$ & 17.6 & $( \pm 2.8)$ & (15.3 to 19.9$)$ & 1.000 & 16.8 & $( \pm 1.2)$ & (16.1 to 17.6$)$ & 0.623 & 17.7 & $( \pm 1.8)$ & (16.8 to 18.7$)$ & 0.995 & 0.150 \\
\hline $\mathbf{A}^{\prime}-\mathbf{M s}$ & 24.3 & $( \pm 1.3)$ & (23.6 to 25.1$)$ & 23.6 & $( \pm 1.4)$ & (22.4 to 24.8$)$ & 0.739 & 23.0 & $( \pm 2.3)$ & (21.5 to 24.4$)$ & 0.208 & 23.3 & $( \pm 2.3)$ & (22.0 to 24.5$)$ & 0.352 & 0.783 \\
\hline Is-Is' & 25.5 & $( \pm 1.8)$ & (24.5 to 26.5$)$ & 25.7 & $( \pm 2.0)$ & (24.0 to 27.4$)$ & 0.990 & 24.1 & $( \pm 2.3)$ & (22.6 to 25.6$)$ & 0.161 & 25.1 & $( \pm 1.5)$ & (24.3 to 25.9$)$ & 0.903 & 0.485 \\
\hline Mo-Ms & 18.6 & $( \pm 1.7)$ & (17.6 to 19.6$)$ & 19.0 & $( \pm 1.9)$ & (17.4 to 20.6$)$ & 0.887 & 17.8 & $( \pm 1.2)$ & (17.0 to 18.6$)$ & 0.376 & 18.1 & $( \pm 1.2)$ & (17.5 to 18.8$)$ & 0.702 & 0.289 \\
\hline Is-Mo & 24.0 & $( \pm 1.3)$ & (23.3 to 24.8$)$ & 23.6 & $( \pm 2.6)$ & (21.4 to 25.8$)$ & 0.942 & 23.3 & $( \pm 2.3)$ & (21.8 to 24.8$)$ & 0.734 & 23.0 & $( \pm 2.1)$ & (21.9 to 24.1$)$ & 0.417 & 0.543 \\
\hline Gn-Cd & 89.7 & $( \pm 3.6)$ & (87.6 to 91.8$)$ & 94.5 & $( \pm 5.4)$ & (89.9 to 99.0$)$ & $0.028^{*}$ & 93.2 & $( \pm 4.0)$ & (90.6 to 95.7$)$ & 0.089 & 90.3 & $( \pm 3.7)$ & (88.4 to 92.3$)$ & 0.956 & 0.924 \\
\hline Pog'-Go & 59.3 & $( \pm 3.0)$ & (57.6 to 61.0$)$ & 61.3 & $( \pm 3.2)$ & (58.6 to 63.9$)$ & 0.309 & 60.7 & $( \pm 2.4)$ & $(59.2$ to 62.2$)$ & 0.486 & 59.6 & $( \pm 3.1)$ & (58.0 to 61.3$)$ & 0.980 & 0.331 \\
\hline Cd-Go & 44.2 & $( \pm 3.0)$ & (42.5 to 45.9$)$ & 44.5 & $( \pm 2.8)$ & (42.2 to 46.9$)$ & 0.989 & 42.2 & $( \pm 2.7)$ & (40.4 to 43.9 ) & 0.176 & 42.3 & $( \pm 2.8)$ & (40.8 to 43.8$)$ & 0.184 & 0.573 \\
\hline Ii-Ii' & 34.2 & $( \pm 1.6)$ & (33.3 to 35.1$)$ & 36.5 & $( \pm 1.9)$ & (34.8 to 38.1$)$ & 0.064 & 33.5 & $( \pm 2.3)$ & (32.0 to 35.0 ) & 0.767 & 35.3 & $( \pm 2.7)$ & (33.9 to 36.7$)$ & 0.404 & 0.978 \\
\hline Mo-Mi & 27.9 & $( \pm 2.1)$ & (26.7 to 29.1$)$ & 29.0 & $( \pm 1.5)$ & (27.8 to 30.3$)$ & 0.499 & 26.8 & $( \pm 1.9)$ & (25.7 to 28.0$)$ & 0.456 & 29.0 & $( \pm 2.6)$ & (27.7 to 30.4$)$ & 0.347 & 0.695 \\
\hline Ii-Mo & 21.1 & $( \pm 1.3)$ & (20.4 to 21.9$)$ & 21.9 & $( \pm 1.8)$ & $(20.5$ to 23.4$)$ & 0.502 & 21.8 & $( \pm 2.1)$ & (20.4 to 23.1$)$ & 0.603 & 21.1 & $( \pm 1.3)$ & (20.4 to 21.8$)$ & 1.000 & 0.378 \\
\hline
\end{tabular}

$2{ }^{*} P<0.05$ by Dunnett' s multiple comparison test. SD, standard deviation. 95\% CI, 95\% Confidence Interval. 


\section{Table 3(on next page)}

Relationships of craniofacial morphology with the differences in cleft type and surgeons

(A) Patient distribution according to the cleft type (B) Patient distribution according to the operating surgeon 
1 Table 3 Relationships of craniofacial morphology with the differences in cleft type and surgeons 2

3 (A) Patients distribution according to the cleft type

\begin{tabular}{|c|c|c|c|}
\hline & $\begin{array}{c}\text { Cleft in hard \& } \\
\text { soft palate }\end{array}$ & Cleft in soft palate & Total \\
\hline Cluster A & 3 & 5 & 8 \\
\hline Cluster B & 9 & 3 & 12 \\
\hline Cluster C & 11 & 5 & 16 \\
\hline Total & 23 & 13 & 36 \\
\hline
\end{tabular}

4

$P\left(\chi^{2}\right)=0.27$, Effect Size; $w=0.457$, Post-hoc power; $(1-\beta)=0.725$

5

6 (B) Patients distribution according to the operating surgeon

\begin{tabular}{|c|c|c|c|c|c|}
\hline & Surgeon A & Surgeon B & Surgeon C & Surgeon D & Total \\
\hline Cluster A & 3 & 1 & 1 & 3 & 8 \\
\hline Cluster B & 4 & 2 & 4 & 2 & 12 \\
\hline Cluster C & 5 & 6 & 2 & 3 & 16 \\
\hline Total & 12 & 9 & 7 & 8 & 36 \\
\hline
\end{tabular}

7

$P\left(\chi^{2}\right)=0.69$, Effect Size; $w=0.775$, Post-hoc power; $(1-\beta)=0.957$ 